\title{
miR-448 downregulates MPPED2 to promote cancer proliferation and inhibit apoptosis in oral squamous cell carcinoma
}

\author{
LINHAN SHEN $^{1 *}$, LIU LIU $^{1 *}$, LIANGYU GE $^{1}$, LONG XIE $^{2}$, SIYU LIU $^{1}$, \\ LEI SANG ${ }^{3}$, TIANTIAN ZHAN ${ }^{1}$ and HONGWEI LI ${ }^{1,4}$ \\ ${ }^{1}$ Jiangsu Key Laboratory of Oral Diseases, Institute of Stomatology, Affiliated Hospital of Stomatology, \\ Nanjing Medical University, Nanjing, Jiangsu 210029; ${ }^{2}$ Department of Oral and Maxillofacial Surgery, \\ Hospital of Stomatology, Wuhan University, Wuhan, Hubei 430079; ${ }^{3}$ Department of Oral and Maxillofacial Surgery, \\ Suzhou Huaxia Stomatological Hospital, Suzhou, Jiangsu 215002; ${ }^{4}$ Jiangsu Key Laboratory of Oral Diseases, \\ Department of Oral and Maxillofacial Surgery, Affiliated Hospital of Stomatology, \\ Nanjing Medical University, Nanjing, Jiangsu 210029 P.R. China
}

Received June 16, 2015; Accepted July 15, 2016

DOI: $10.3892 / \mathrm{etm} .2016 .3659$

\begin{abstract}
The incidence of oral squamous cell carcinoma (OSCC) is continuously increasing while its survival rate has not notably improved. There is a pressing need for improved understanding of the genetic regulation of OSCC tumorigenesis and progression. In this study, the function of miR-448 in the regulation of OSCC growth and its putative target were thoroughly analyzed in vitro. The expression of miR-448 was detected in human OSCC specimens and OSCC cell lines (Cal-27 and Scc-9) by reverse transcription-quantitative polymerase chain reaction. The function of miR-448 was investigated in Cal-27 cells transfected with miR-448 inhibitor, and its putative target determined using a luciferase reporter assay. MTT and wound healing assays and flow cytometry were used to evaluate the effects of miR-448 on OSCC proliferation, metastasis and apoptosis. The level of miR-448 was significantly elevated in human OSCC tissues and the Cal-27 cell line. Suppression of miR-448 expression attenuated cell proliferation and migration, and induced apoptosis of Cal-27 cells. Furthermore, miR-448 bound with the 3'-untranslated region of metallophosphoesterase domain containing 2 (MPPED2) mRNA, thereby reducing the MPPED2 protein level. Thus, it appears that miR-448 acts as a tumor inducer, causing OSCC growth by inhibiting the expression of its target MPPED2.
\end{abstract}

Correspondence to: Professor Hongwei Li, Jiangsu Key Laboratory of Oral Diseases, Department of Oral and Maxillofacial Surgery, Affiliated Hospital of Stomatology, Nanjing Medical University, 136 Hanzhong Road, Nanjing, Jiangsu 210029, P.R. China

E-mail: lhwqhxa@sina.com

${ }^{*}$ Contributed equally

Key words: oral squamous cell carcinoma, miR-448, MPPED2
These results demonstrate that miR-448 plays a critical role in OSCC tumorigenesis, and is a potential therapeutic target.

\section{Introduction}

Oral squamous cell carcinoma (OSCC) is the sixth most common cancer worldwide, and accounts for $>40 \%$ of head and neck malignancies (1). Despite advances in treatment and diagnostics, the incidence and mortality rates associated with OSCC are increasing (2). Metastasis and recurrence of OSCC are significant factors $(3,4)$. As with other cancers, the accumulation of genetic and epigenetic changes is involved in the development and progression of OSCC (5). Therefore, there is an urgent requirement to study these genetic changes in order to deliver identify diagnostic or biomarkers, and therapeutic targets for early detection and prognosis.

MicroRNAs (miRNAs) are endogenous non-coding RNAs of 22 nucleotides, and are evolutionarily conserved (6). They regulate post-transcriptional gene expression through binding to specific regions of target mRNA in a sequence-specific manner (6). It is now clear that miRNAs play crucial roles in various biological processes such as differentiation, proliferation, invasion and apoptosis (7). Moreover, increasing evidence has indicated that miRNAs are involved in the pathogenesis of various human cancers by serving as either oncogenes or tumor suppressor genes $(8,9)$. Several miRNAs, such as miR-31 and miR-99a, have been shown to be dysregulated in OSCC and contribute to the development and progression of OSCC $(10,11)$. miR-448 is reportedly associated with various human malignancies. It is upregulated in breast cancer and early cerebral aneurysm $(12,13)$, indicating regulatory effects of miR-448 in various conditions.

In the present study, the expression levels of miR-448 were investigated in OSCC cancer cell lines and OSCC cancer tissues from patients. Moreover, an miR-448 inhibitor was transfected into an OSCC cell line, and the effects of miR-448 on cancer-related growth, migration and anti-apoptosis were analyzed. In addition, the involvement of the predicted target 
of miR-488, metallophosphoesterase domain containing 2 (MPPED2), in miR-448 activity in OSCC cells was investigated. The results of this study may provide further insights into the mechanisms responsible for OSCC tumorigenesis, and indicate whether miR-448 is a potential therapeutic target in OSCC treatment.

\section{Materials and methods}

Clinical specimens and cell culture. Fresh cancer tissues and matched adjacent non-cancerous tissues were obtained from 15 patients with OSCC at Nanjing Stomatological Hospital (Nanjing, China) between November 2013 and February 2014. The samples were snap-frozen in liquid nitrogen in the operating room and stored at $-80^{\circ} \mathrm{C}$ until extraction of total RNA. Written informed consent was obtained from all patients and the study was approved by the Institutional Review Board of the Affiliated Hospital of Stomatology, Nanjing Medical University. Human OSCC cell lines SCC-9 and Cal-27 were cultured with complete medium containing $10 \%$ fetal bovine serum (Hyclone; GE Healthcare Life Sciences, Logan, UT, USA) and Dulbecco's modified Eagle's medium (DMEM)-F12 (Hyclone; GE Healthcare Life Sciences) at $37^{\circ} \mathrm{C}$ with $5 \%$ $\mathrm{CO}_{2}$. The medium was changed every 2 days. Cell lines were provided by Shanghai Ninth People's Hospital Affiliated Shanghai Jiaotong University School of Medicine (Shanghai, China).

Reverse transcription-quantitative polymerase chain reaction (RT-qPCR). Total RNA was extracted from OSCC cancer tissues and cells using TRIzol reagent (Invitrogen; Thermo Fisher Scientific, Inc., Waltham, MA, USA), according to the manufacturer's protocol. The RNA was digested with RQ1 RNase-Free DNase (M6101; Promega Corporation, Madison, WI, USA) for $30 \mathrm{~min}$ to remove genomic contaminants. Subsequently, DNase was removed with an RNeasy Mini kit (Qiagen GmbH, Hilden, Germany). RNA was quantified using a NanoDrop ND-1000 Spectrophotometer (Thermo Fisher Scientific, Inc., Wilmington, DE, USA). The RNA was then transcribed into cDNA using a Mir- ${ }^{\mathrm{TM}}$ miRNA First-Strand Synthesis kit (cat. no. 638313; Takara Bio, Inc., Otsu, Japan), following the manufacturer's protocol. Briefly, the transcription was performed in a $10-\mu 1$ reaction mixture, containing $5 \mu 1 \mathrm{mRQ}$ Buffer (2X), $3 \mu 1 \mathrm{RNA}$ sample and $1.0 \mu 1 \mathrm{mRQ}$ Enzyme. Reaction was conducted for $1 \mathrm{~h}$ at $37^{\circ} \mathrm{C}$, and then terminated by heating at $85^{\circ} \mathrm{C}$ for $5 \mathrm{~min}$ to inactivate the

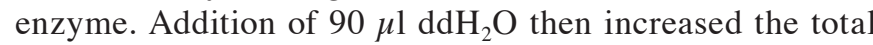
volume to $100 \mu \mathrm{l}$.

qPCR was then performed on a 7500 Real-Time-PCR System (Applied Biosystems; Thermo Fisher Scientific, Inc.) using a Mir- ${ }^{\mathrm{TM}}$ miRNA qRT-PCR SYBR ${ }^{\circledR}$ kit (Takara Bio, Inc.). The experiment was performed in a $25-\mu 1$ reaction mixture, containing $9 \mu 1 \mathrm{DDH}_{2} 0,12.5 \mu \mathrm{l} \mathrm{SYBR}$ Advantage Premix (2X), $0.5 \mu 1$ ROX Dye (50X), $0.5 \mu 1$ miRNA-specific Primer $(10 \mu \mathrm{M}), 0.5 \mu \mathrm{lmRQ} 3^{\prime}$ Primer 0.5 and $2.0 \mu \mathrm{l} \mathrm{cDNA}$. PCR was then performed with the following cycling profile: $95^{\circ} \mathrm{C}$ for $5 \mathrm{sec}$, followed by 30 cycles of $60^{\circ} \mathrm{C}$ for $30 \mathrm{sec}$. Data were normalized to the internal control, $\mathrm{U} 6$, to obtain $\Delta \mathrm{Ct}$. The final value of the gene of interest relative to the untreated cells was converted by the $2^{-\Delta \Delta C T}$ method (14). Primers used were as follows: miR-448 forward: 5'-TTGCATATGTAG GATGTCCCAT-3' and reverse: 5'-CTCAACTGGTGTCGT GGAGTCGGCAATTCAGTTGAGATGGGACA-3'; U6 forward: 5'-CGCTTCGGCAGCACATATAC-3' and reverse: 5'-ACGAATTTGCGTGTCATCCT- 3'. The mRQ 3' Primer was provided with the Mir- ${ }^{\mathrm{TM}}$ miRNA qRT-PCR $\mathrm{SYBR}^{\circledR}{ }^{\circledR}$ kit. All samples were analyzed in triplicate.

Western blot analysis. Total protein was extracted from cells with lysis buffer (Bio-Rad Laboratories, Inc., Hercules, CA, USA). The lysates were analyzed using a BCA protein assay kit (Pierce Biotechnology, Inc., Rockford, IL, USA). Proteins (20 $\mu 1)$ were subjected to sodium dodecyl sulfate-polyacrylamide gel electrophoresis with $10 \%$ polyacrylamide gels and transferred to polyvinylidene fluoride membranes. These were blocked with $5 \%$ non-fat milk for $2 \mathrm{~h}$ at room temperature, prior to incubation with primary antibodies against MPPED2 (1:500; ab87077; Abcam, Cambridge, UK) at $4^{\circ} \mathrm{C}$ overnight. The membranes were washed three times and incubated with horseradish peroxidase (HRP)-conjugated IgG secondary antibody (1,1000; BA1055; Wuhan Boster Biological Technology, Ltd., Wuhan, China) at room temperature for $2 \mathrm{~h}$. Subsequently, protein bands were detected by enhanced chemiluminescence (Immobilon ${ }^{\mathrm{TM}}$ Western Chemiluminescent HRP Substrate; WBKLS0100; EMD Millipore, Billerica, MA, USA) and visualized using a n ImageQuantLAS 4000 mini imaging system (GE Healthcare Life Sciences, Chicago, IL, USA). Protein levels were normalized to $\beta$-actin, using a rabbit monoclonal anti- $\beta$-actin antibody (1:100; BM0627; Wuhan Boster Biological Technology, Ltd., Wuhan, China).

Plasmids and transfection. The miR-448 inhibitor (miR-448-in) and negative control (NC-in) were designed and synthesized by Shanghai GenePharma Co., Ltd. (Shanghai, China). OSCC cells were grown in 6-well plates to $60 \%$ confluence prior to transfection. The transfection procedure was performed using Lipofectamine 2000 in accordance with the manufacturer's protocol (Invitrogen; Thermo Fisher Scientific, Inc.). Total RNA was extracted at $48 \mathrm{~h}$ post-transfection and used for RT-qPCR analysis.

Cell proliferation assay. Cell proliferation activity was examined with the 3-(4,5-dimethylthiazol-2-yl)-2,5-diphenyltetrazolium bromide (MTT) assay. At $6 \mathrm{~h}$ after transfection, cells in three groups [blank (untransfected), miR-448-in and NC-in] were digested and seeded into a 96-well microtiter plate for $0,24,48$ and $72 \mathrm{~h}$. MTT solution $(10 \mu \mathrm{l})$ was added to each well at the above time points. The optical density (OD) values were measured at $570 \mathrm{~nm}$ using a microplate reader (Labsystems, Santa Fe, NM, USA). Each experimental condition was tested three times and average results were calculated.

Wound healing assay. Cell migration was evaluated using a scratched wound healing assay conducted with plastic plate wells. When the cell confluence reached $\sim 80 \%$ (at $\sim 48 \mathrm{~h}$ post-transfection), scratch wounds were made by scraping the cell layer across each culture plate using the tip of a 10- $\mu 1$ pipette. After wounding, the debris was removed by washing the cells with phosphate-buffered saline (PBS). Three wounds were made for each sample, and photographic images of the 
A

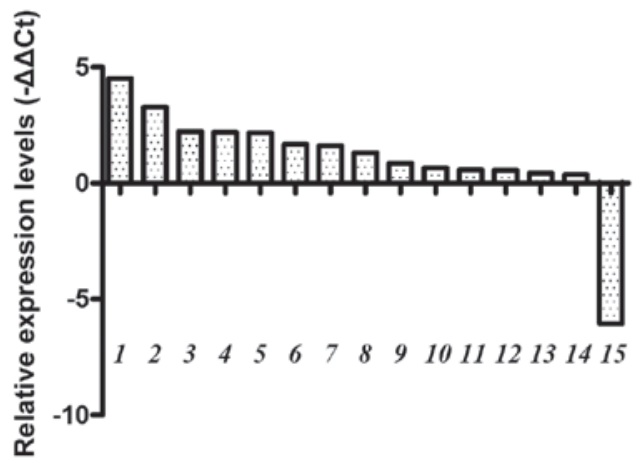

B

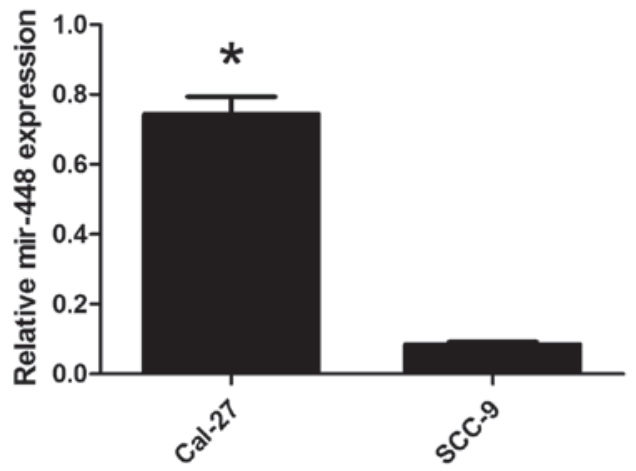

Figure 1. Relative miR-448 expression in OSCC tissues and cell lines. (A) RT-qPCR results of miR-448 in 15 pairs of OSCC cancer tissues and adjacent tissues, showing higher expression in 14 samples of OSCC cancer tissues than in the adjacent tissues ( $\mathrm{P}<0.05$ vs. control). (B) RT-qPCR results of miR-448 expression in OSCC cell lines. * $\mathrm{P}<0.05$ vs. SCC-9. RT-qPCR, reverse transcription-quantitative polymerase chain reaction; OSCC, oral squamous cell carcinoma.

wound were captured at $0 \mathrm{~h}$ and subsequent time points $(24$, 36 and 48 h). Cell migration was evaluated by measuring the width of the wound at the same position. The experiments were performed in triplicate.

Measurement of apoptosis by flow cytometry. OSCC cancer cells were grown and transfected as above. For apoptotic analysis, the transfection group and control group were seeded in a 6-well culture plate. After $48 \mathrm{~h}$ of culture, cells including those suspended in the medium were collected, washed with PBS and resuspended in $1 \mathrm{X}$ binding buffer at a concentration of $1 \times 10^{5}$ cells $/ \mathrm{ml}$. Annexin-V-allophycocyanin (APC) $(5 \mu \mathrm{l})$ and 7-aminoactinomycin D (7-AAD; $5 \mu \mathrm{l})$ were added to the cell suspension and the suspension was incubated for $15 \mathrm{~min}$ in the dark. Cell apoptosis was assessed by flow cytometry (Becton Dickinson, San Jose, CA, USA).

Target prediction. Three online programs, miRanda (http://www.microrna.org/microrna/home.do), TargetScan (http:www.targetscan.org), and TarBase (http://diana.imis. athena-innovation.gr/DianaTools/index.php?r=tarbase/index) were used in combination with previous reports, for predicting the target gene of miR-448.

Vector construction and luciferase reporter assay. The 3' untranslated region (3'-UTR) of the human MPPED2 sequence (the predicted target) was amplified by PCR from human genomic DNA using the following primers: Forward (F), 5'-CTTGACTCGAGAGCTCTAAATGCCCTATTGG-3' and reverse (R), 5'-ATTGCGGCCGCGTGGGTAATAAAAATT TATTG-3'. Mutant 3'-UTRs of MPPED2 were obtained by overlapping PCR using the following primers: m1-F, 5'-GCC TTTATATtaAAATAAAATTGC-3'; m1-R 5'-GCAATTTTA TTTtaATATAAAGGC-3'; m2-F 5'-GATTTATTCATATtaAACATCAGTA-3'; m2-R, 5'-TACTGATGTTtaATATGAATA AATC-3' (mutations are indicated by lower case letters). Each sequence was cloned into the pmiR-RB-REPORT ${ }^{\mathrm{TM}}$ vector (Promega Corporation, Madison, WI, USA) and the construct was verified by sequencing. A luciferase assay was conducted using a Dual-Luciferase Reporter Assay System (ZZE1980; Promega Corporation) according to the manufacturer's instructions. Renilla luciferase was cotransfected into the cells as a control for normalization.

Statistical analysis. Statistical analysis was performed using SPSS version 13.0 software (SPSS, Inc., Chicago, IL, USA). Data are expressed as the mean \pm standard deviation from at least three separate experiments. Differences between groups were analyzed using a Student's t-test. $\mathrm{P}<0.05$ was considered to indicate a statistically significant difference.

\section{Results}

Expression of miR-448 in OSCC tissues and cells. The expression patterns of miR-448 were determined by RT-qPCR analysis in 15 pairs of OSCC and matched adjacent non-cancerous oral tissues. As shown in Fig. 1A, the expression levels of miR-448 were clearly upregulated in OSCC tissues compared with the levels in non-cancerous oral tissues. The expression of miR-448 was also detected in the OSCC cell lines Cal-27 and SCC-9. RT-qPCR indicated that the expression level of miR-448 was higher in the Cal-27 cell line than in the SCC-9 cell line (Fig. 1B). These results suggest that miR-448 might be involved in the development of OSCC in humans. The difference between the miR-488 levels in the Cal-27 and SCC-9 cell lines may be associated with differences in the aggressiveness of these tumors.

Silencing of miR-448 inhibits cell growth in vitro. To assess the effect of miR-448 on the biological properties of OSCC cancer cells, miR-448 inhibitor (miR-448-in) or negative control (NC-in) was transfected into Cal-27 cells. The knock-down of the expression level of miR-448 was verified by RT-qPCR (Fig. 2A). Subsequently, the effect of miR-448 on the proliferation of OSCC cells was examined using an MTT assay. It was observed that the viability of the cells was reduced by the inhibition of miR-448, suggesting that miR-448 promotes the proliferation of Cal-27 cells (Fig. 2B). These results suggest a growth-promoting role of miR-448 in OSCC.

miR-448 inhibition significantly suppresses the migration of Cal-27 cells in vitro. A wound healing assay was used to observe changes in tumor migration ability. The results showed 
A

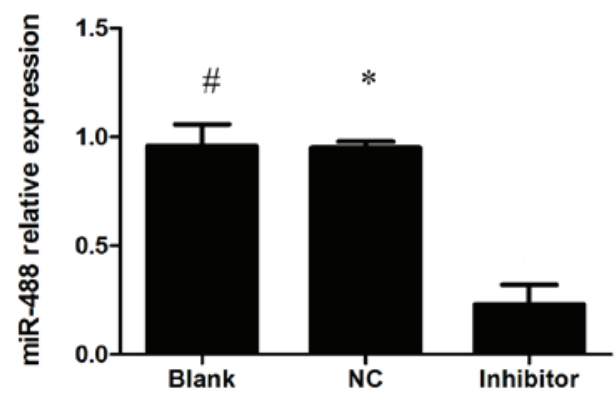

B

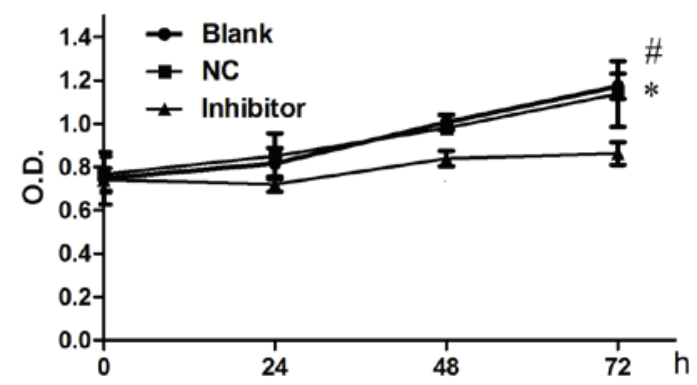

Figure 2. miR-448 promotes the proliferation of Cal-27 cells. (A) Expression levels of miR-448 were tested by RT-qPCR in Cal-27 cells transfected with miR-448 inhibitor (miR-448-in) or negative control (NC-in). (B) MTT assay results for the transfected cell lines. " $\mathrm{P}<0.05$ for NC vs. inhibitor; ${ }^{*} \mathrm{P}<0.05$ for blank vs. inhibitor. RT-qPCR, reverse transcription-quantitative polymerase chain reaction; OSCC, oral squamous cell carcinoma; MTT, 3-(4,5-dimethylthiazol-2-yl)-2,5-diphenyltetrazolium bromide; O.D., optical density.

A

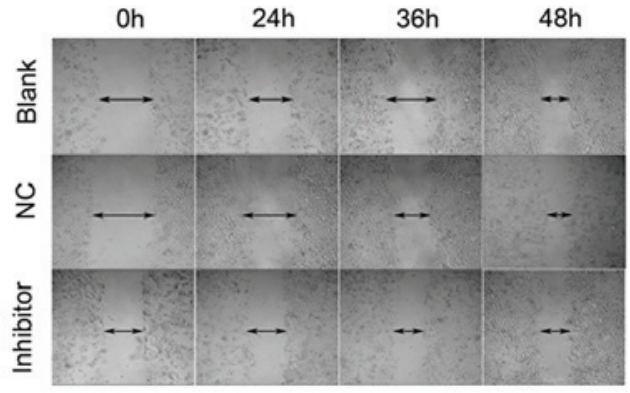

Blank

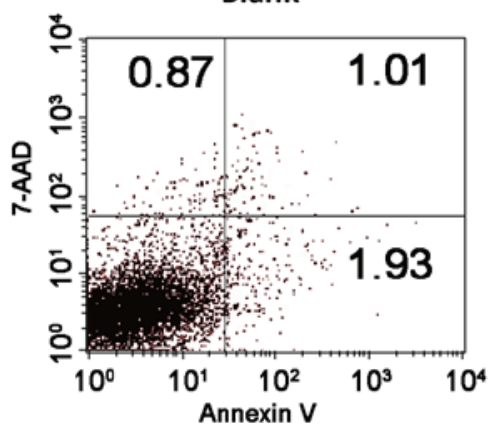

NC

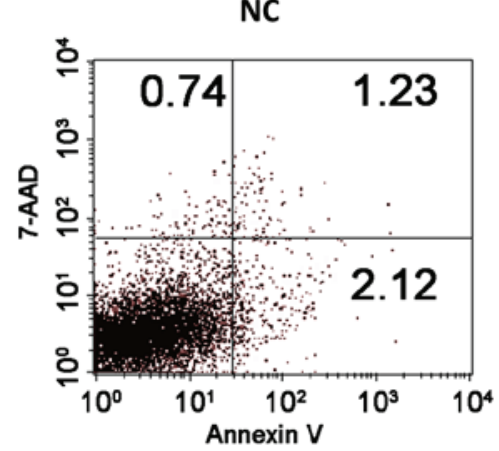

Inhibitor

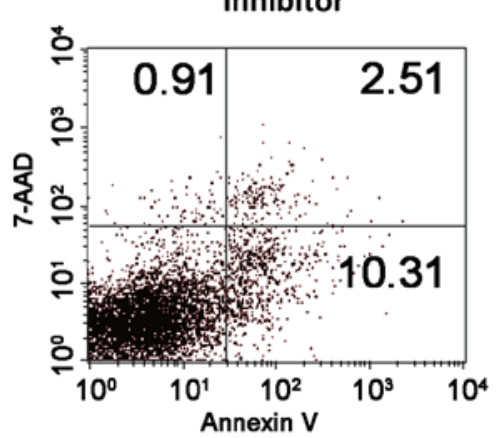

Figure 4. Apoptosis assay results. The percentage of apoptotic cells in the total measured cell population is shown in the upper right quadrant. A representative experiment of three performed is shown for each group. NC, normal control; 7-AAD, 7-aminoactinomycin D.

that following transfection into Cal-27 cells, the miR-448 inhibitor reduced the ability of the cells to migrate (Fig. 3).

miR-448 reduces the apoptosis of OSCC cells. To evaluate the effect of miR-448 on OSCC cell apoptosis, apoptosis was measured at $48 \mathrm{~h}$ after NC or miR-448 inhibitor transfection

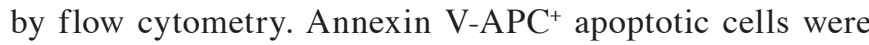
markedly increased in the miR-448 inhibitor-transfected group compared with the NC or blank control groups. The percentage of apoptotic cells in the group transfected with miR-448 inhibitor was higher than that of the control groups (Fig. 4). The findings indicate an anti-apoptotic role for miR-448 in OSCC cells.
miR-448 directly inhibits the expression of MPPED2 by binding to the 3'-UTR. Bioinformatics analysis identified that MPPED2 is target of miR-448 having a close association with miR-448. The predicted binding sites between miR-448 and the 3'-UTR of MPPED2 are illustrated in Fig. 5A. To explore the association between MPPED2 and miR-448, qPCR and western blot analysis was used to measure the change of MPPED2 expression that occurred when miR-488 was inhibited. The results showed that the miR-448 inhibitor increased MPPED2 expression at the mRNA and protein levels, indicating that miR-488 reduces MPPED2 expression (Fig. 5B and C). To determine if the suppressive effects of miR-448 on MPPED2 were achieved via direct action, fragments 
A

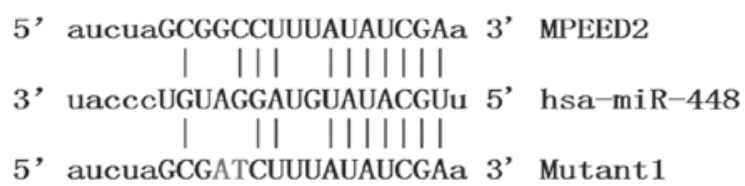

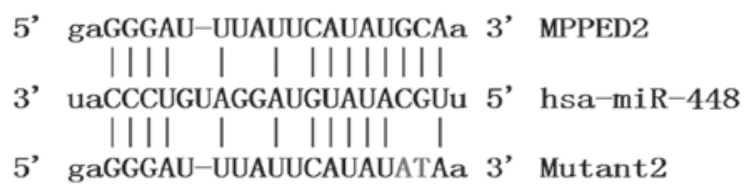

C

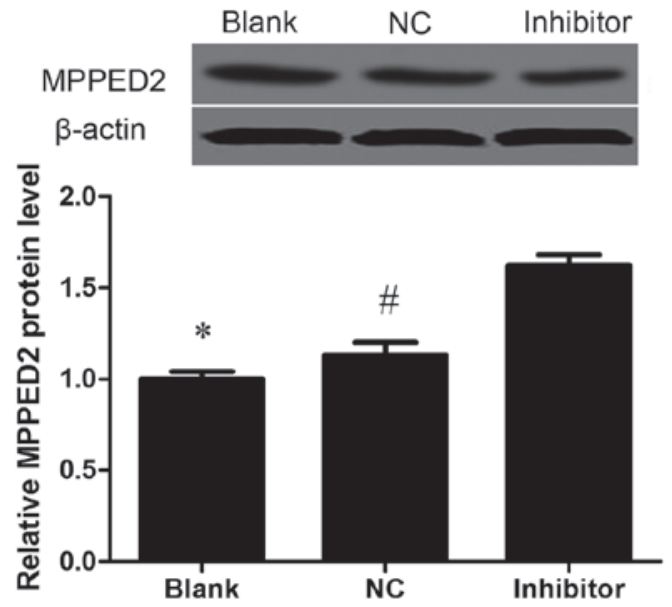

B

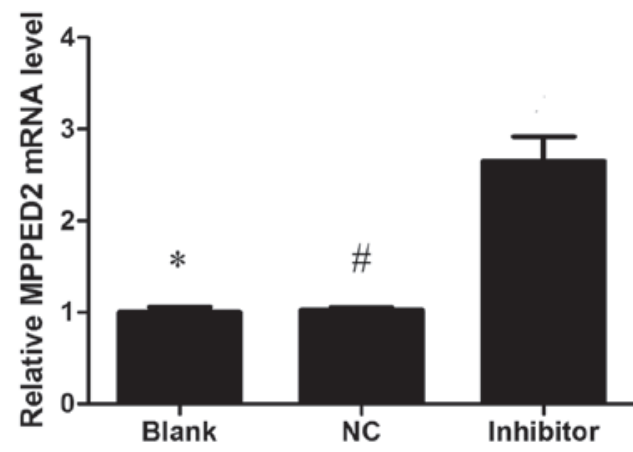

D

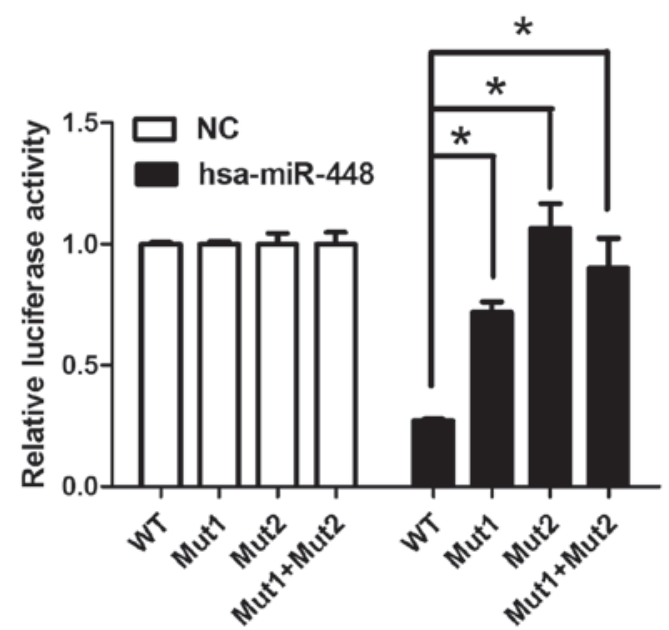

Figure 5. miR-448 targets the MPPED2 gene. (A) Putative binding site of miR-448 in the 3'-UTR of MPPED2 (as detected by TargetScan). Only matched nucleotides with miRNA seed sequences are indicated with vertical lines. MPPED2 expression in Cal-27 cells transfected with NC or miR-448-inhibitor as determined by (B) reverse transcription-quantitative polymerase chain reaction analysis and (C) western blotting. MPPED2 levels are significantly higher in the inhibitor group than in the blank $(" \mathrm{P}<0.05)$ and $\mathrm{NC}\left({ }^{*} \mathrm{P}<0.05\right)$ groups. (D) Wild-type (WT) or mutant (Mut) reporter plasmids were cotransfected with miR-448 or NC into Cal-27 cells. Luciferase activity determined $48 \mathrm{~h}$ after transfection. " $\mathrm{P}<0.05$. MPPED2, metallophosphoesterase domain containing 2; UTR, untranslated region; NC, normal control.

containing the miR-448 binding sites of wild-type and mutant 3'-UTRs of MPPED2 were subcloned into a luciferase reporter vector. As shown in Fig. 5D, miR-448 suppressed MPPED2 luciferase activities in Cal-27 cells, and this suppression of activity was abrogated by mutations in the miR-448 binding sites, suggesting that miR-448 directly targets MPPED2.

\section{Discussion}

OSCC is a health problem worldwide with increasing incidence and mortality rates. Approximately 300,000 patients are estimated to have oral cancer worldwide annually $(15,16)$. Previous studies have identified that the occurrence and development of OSCC correlates with various molecules $(17,18)$. MicroRNAs have been found to be differentially altered in various tumors and might contribute to the activation and upregulation of key molecules in cancers (19-21). It may assist us to identify predictive marker of prognosis and potential treatment target to investigate the effective molecular expression in OSCC $(22,23)$. Despite evidence indicating the regulatory role of microRNAs in cancer progression and invasion, their regulatory role in oral cancer metastasis remains poorly understood.
miR-448 has been reported to be associated with various types of cancer. It is upregulated in breast cancer (24) and early cerebral aneurysm (12), and downregulated in hepatocellular carcinoma (25) . It has also been reported that miR-448 is the most downregulated microRNA following chemotherapy in breast cancer (13), suggesting that it may act as an oncogene or anti-oncogene in different cancers. The results of the present study suggest that the expression of miR-448 in OSCC tissues was significantly higher than that in matched paraneoplastic normal tissues. The molecular mechanisms underlying the upregulation in OSCC remain unclear. It may be speculated that miR-448 has an association with the occurrence and development of OSCC. The results of the present study demonstrated that miR-448 was upregulated in OSCC tissues and cell lines. Moreover, they indicate that miR-448 promotes the proliferation and migration of OSCC cells in vitro, suggesting an oncogenic role in OSCC..

The molecular mechanisms by which miR-448 promotes the proliferation and migration of OSCC cells were further investigated in the present study. Publicly available bioinformatic algorithms predicted MPPED2 as a theoretical target gene of miR-448. MPPED2 has been recognized to have a role in brain development and tumorigenesis (26). Also 
MPPED2 has been found to be significantly associated with caries via meta-analysis (27). MPPED2 is downregulated in oral epithelial cells in response to oral pathogens (28). These observations encourage further research into MPPED2. The results of the present study indicated that MPPED2 expression was suppressed by miR-448 in OSCC cells, while inhibition of miR-448 rescued the expression of MPPED2. Luciferase reporter assays revealed that miR-448 directly targeted the 3'-UTR of MPPED2 mRNA. These results suggest that miRNA-448 promotes the proliferation of OSCC cells by negatively regulating the expression of MPPED2 via directly targeting the 3 '-UTR of this gene. Although the detailed molecular mechanisms of miR-448 in OSCC have not been clearly clarified, these results imply that miR-448, as a tumor promoting microRNA, could regulate cancer cell proliferation and metastasis in OSCC. These results might help to elucidate the cause and effects of altered expression of miR-448 in the initiation and progression of OSCC in the future.

In summary, the present study has demonstrated that miR-448 expression is significantly upregulated in OSCC tissues and cells. It also confirmed that miR-448 negatively regulates MPPED2 transcription and translation through directly binding to the 3'-UTR of this gene. Furthermore, the study demonstrated that the repression of miR- 448 reduced the proliferation and metastasis of OSCC cells, and thereby indicated that miR-448 induces the proliferation and metastasis of these cells. These results suggest that miR-448 is potentially useful as a biomarker and therapeutic target in OSCC.

\section{Acknowledgements}

This study was supported by a project funded by the Priority Academic Program Development of Jiangsu Higher Education Institutions (PAPD, 2014-37).

\section{References}

1. Warnakulasuriya S: Global epidemiology of oral and oropharyngeal cancer. Oral Oncol 45: 309-316, 2009.

2. Carvalho AL, Singh B, Spiro RH, Kowalski LP and Shah JP: Cancer of the oral cavity: A comparison between institutions in a developing and a developed nation. Head Neck 26: 31-38, 2004

3. Grimm M, Alexander D, Munz A, Hoffmann J and Reinert S: Increased LDH5 expression is associated with lymph node metastasis and outcome in oral squamous cell carcinoma. Clin Exp Metastasis 30: 529-540, 2013.

4. Olasz L, Orsi E, Markó T and Szalma J: Induction chemotherapy response and recurrence rates in correlation with $\mathrm{N} 0$ or $\mathrm{N}+$ stage in oral squamous cell cancer (OSCC). Cancer Metastasis Rev 29: 607-611, 2010.

5. Kang MK and Park NH: Conversion of normal to malignant phenotype: Telomere shortening, telomerase activation and genomic instability during immortalization of human oral keratinocytes. Crit Rev Oral Biol Med 12: 38-54, 2001.

6. Liao WT, Li TT, Wang ZG, Wang SY, He MR, Ye YP, Qi L, Cui YM, Wu P, Jiao HL, et al: MicroRNA-224 promotes cell proliferation and tumor growth in human colorectal cancer by repressing PHLPP1 and PHLPP2. Clin Cancer Res 19: 4662-4672, 2013.

7. Ambros V: The functions of animal microRNAs. Nature 431: 350-355, 2004

8. Calin GA, Sevignani C, Dumitru CD, Hyslop T, Noch E, Yendamuri S, Shimizu M, Rattan S, Bullrich F, Negrini M and Croce CM: Human microRNA genes are frequently located at fragile sites and genomic regions involved in cancers. Proc Natl Acad Sci USA 101: 2999-3004, 2004.
9. Iorio MV and Croce CM: MicroRNAs in cancer: Small molecules with a huge impact. J Clin Oncol 27: 5848-5856, 2009.

10. Hung PS, Tu HF, Kao SY, Yang CC, Liu CJ, Huang TY, Chang KW and Lin SC: MiR-31 is upregulated in oral premalignant epithelium and contributes to the immortalization of normal oral keratinocytes. Carcinogenesis 35: 1162-1171, 2014.

11. Yen YC, Shiah SG, Chu HC, Hsu YM, Hsiao JR, Chang JY, Hung WC, Liao CT, Cheng AJ, Lu YC and Chen YW: Reciprocal regulation of microRNA-99a and insulin-like growth factor I receptor signaling in oral squamous cell carcinoma cells. Mol Cancer 13: 6, 2014.

12. Jeong SH, Lee HJ, Yi JS, Lee HJ, Lee IW, Park KC and Yang JH: Identification of microRNAs with altered expression profiles in a rat model of experimentally induced early cerebral aneurysms. Korean J Neurotrauma 9: 41, 2013.

13. Li QQ, Chen ZQ, Cao XX, Xu JD, Xu JW, Chen YY, Wang WJ, Chen Q, Tang F, Liu XP and Xu ZD: Involvement of NF- $\mathrm{NB} / \mathrm{miR}-448$ regulatory feedback loop in chemotherapy-induced epithelial-mesenchymal transition of breast cancer cells. Cell Death Differ 18: 16-25, 2011.

14. Livak KJ and Schmittgen TD. Analysis of relative gene expression data using real-time quantitative PCR and the 2(-Delta Delta C(T)) Method. Methods 25: 402-408, 2001.

15. Rautava J, Luukkaa M, Heikinheimo K, Alin J, Grenman R and Happonen RP: Squamous cell carcinomas arising from different types of oral epithelia differ in their tumor and patient characteristics and survival. Oral Oncol 43: 911-919, 2007.

16. Mehrotra R, Singh MK, Pandya S and Singh M: The use of an oral brush biopsy without computer-assisted analysis in the evaluation of oral lesions: A study of 94 patients. Oral Surg Oral Med Oral Pathol Oral Radiol Endod 106: 246-253, 2008.

17. Peng CH, Liao CT, Peng SC, Chen YJ, Cheng AJ, Juang JL, Tsai CY, Chen TC, Chuang YJ, Tang CY, et al: A novel molecular signature identified by systems genetics approach predicts prognosis in oral squamous cell carcinoma. PLoS One 6: e23452, 2011.

18. Chen R, Yang K, Zhao NB, Zhao D, Chen D, Zhao CR and Tang H: Abnormal expression of PER1 circadian-clock gene in oral squamous cell carcinoma. Onco Targets Ther 5: 403-407, 2012.

19. Kunej T, Godnic I, Ferdin J, Horvat S, Dove P and Calin GA: Epigenetic regulation of microRNAs in cancer: An integrated review of literature. Mutat Res 717: 77-84, 2011.

20. Jiang YW and Chen LA: MicroRNAs as tumor inhibitors, oncogenes, biomarkers for drug efficacy and outcome predictors in lung cancer (review). Mol Med Rep 5: 890-894, 2012.

21. Perrotti D and Eiring AM: The new role of microRNAs in cancer. Future Oncol 6: 1203-1206, 2010.

22. Lohavanichbutr P, Houck J, Doody DR, Wang P, Mendez E, Futran N, Upton MP, Holsinger FC, Schwartz SM and Chen C: Gene expression in uninvolved oral mucosa of OSCC patients facilitates identification of markers predictive of OSCC outcomes. PLoS One 7: e46575, 2012.

23. Hsu DS, Chang SY, Liu CJ, Tzeng CH, Wu KJ, Kao JY and Yang MH: Identification of increased NBS1 expression as a prognostic marker of squamous cell carcinoma of the oral cavity. Cancer Sci 101: 1029-1037, 2010.

24. Mak KK, Wu AT, Lee WH, Chang TC, Chiou JF, Wang LS, Wu CH, Huang CY, Shieh YS, Chao TY, et al: Pterostilbene, a bioactive component of blueberries, suppresses the generation of breast cancer stem cells within tumor microenvironment and metastasis via modulating NF- $\kappa$ B/microRNA 448 circuit. Mol Nutr Food Res 57: 1123-1134, 2013.

25. Katayama Y, Maeda M, Miyaguchi K, Nemoto S, Yasen M, Tanaka S, Mizushima H, Fukuoka Y, Arii S and Tanaka H. Identification of pathogenesis-related microRNAs in hepatocellular carcinoma by expression profiling. Oncol Lett 4: 817-823, 2012.

26. Pattaro C, Köttgen A, Teumer A, Garnaas M, Böger CA, Fuchsberger C, Olden M, Chen MH, Tin A, Taliun D, et al: Genome-wide association and functional follow-up reveals new loci for kidney function. PLoS Genet 8: e1002584, 2012.

27. Shaffer JR, Wang X, Feingold E, Lee M, Begum F, Weeks DE, Cuenco KT, Barmada MM, Wendell SK, Crosslin DR, et al: Genome-wide association scan for childhood caries implicates novel genes. J Dent Res 90: 1457-1462, 2011.

28. Stanley BO, Feingold E, Cooper M, Vanyukov MM, Maher BS, Slayton RL, Willing MC, Reis SE, McNeil DW, Crout RJ, et al: Genetic association of MPPED2 and ACTN2 with dental caries. J Dent Res 93: 626-632, 2014. 